\title{
A Case of Benign Giant, Schwannoma and Review of literature
}

\author{
Mamun SMA ${ }^{1}$, Haider $\mathrm{Z}^{2}$, Talukder $\mathrm{QI}^{3}$, Zahangir $\mathrm{NM}^{4}$
}

\begin{abstract}
Though variety of benign and malignant tumors of peripheral nerve origin can occur in the mediastinum, benign schwannoma is the most common neurogenic tumor in the mediastinum Schwannoma is a non cancerous (benign) tumor that involves the Schwann cells in the protective covering of nerves, called the myelin sheath. Schwannoma is the common neurogenic tumor in the mediastinum. They are most often asymptomatic but sometimes accompanied by bloody pleural effusion. Herein, we report a case of giant, benign schwannoma presented with minimum symptoms of back pain and occasional chest pain. The case is reported here along with a literature review.
\end{abstract}

\section{Keywords}

Schwannoma, Mediastinum

\section{Introduction}

A variety of benign and malignant tumors of peripheral nerve origin can occur in the mediastinum. They are most frequently found in the posterior compartment of the mediastinum and benign schwannoma is the most common tumor type. Mediastinal benign schwannomas are usually asymptomatic and if symptoms are present they are usually caused by compression of nerve or blood vessel. Benign schwannomas are rarely accompanied by pleural effusion and bloody effusion is usually associated with malignant schwannoma. The authors experienced a case of giant, benign schwannoma which presented with total collapse of right lung by massive, bloody pleural effusion. The case is reported here along with a literature review.

\section{Case Report}

A 46-year-old, active female presented with mild chest discomfort and pain in the back of chest, in the Respiratory Medicine clinic of Apollo Hospitals Dhaka.
Patient also complained of occasional cough but no episode of haemoptysis. She had no constitutional symptom like anorexia, weight loss, and fever. Her physical examination reveals no significant abnormality but diminished breath sound in right lower chest. Chest X-ray (Fig 1)

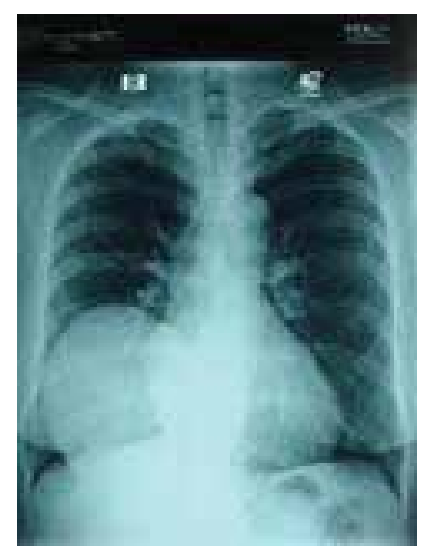

Fig 1: Chest X-ray P/A view before removal of cyst. 
showed a rounded opacity in the lower part of right lung obscuring the right dome of diaphragm. Chest computed tomography (Fig 2) revealed big cystic lesion.

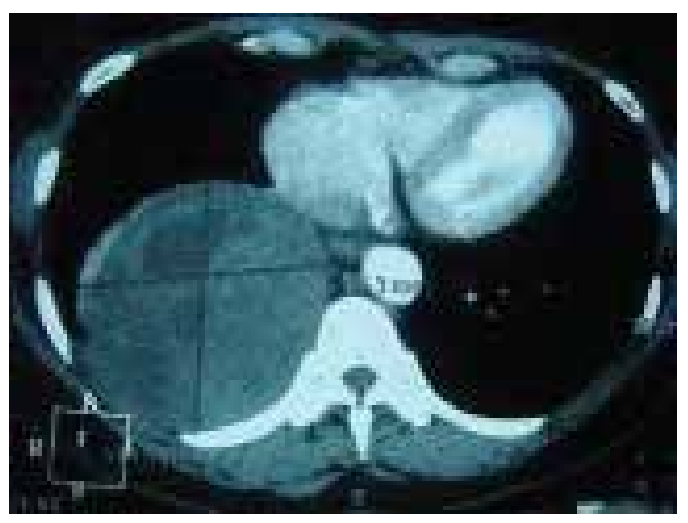

Fig 2: Computed tomography before removal of cyst.

The mass was well-circumscribed and showed minimal enhancement by radio-contrast dye. In lung function, Baseline Spirometry revealed moderate restrictive disorder. Arterial blood gas and CO transfer factor test was normal.
An open thoracotomy, followed by complete resection of the mass was performed. The mass was tightly fixated to the thoracic wall. Upon removal, the mass was revealed to be a whitish soft tumor measuring $10.0 \times 12.0 \times 3.0 \mathrm{~cm}$ with a yellow cut-surface. Upon microscopic exam, the tumor (Fig 3) was composed of spindle cells with elongated nuclei, forming interlacing bundle with focal nuclear palisading. Mitotic figures were rare. Immuno histo chemical studies showed a strongly positive reaction with S-100 protein (data not shown). All of these findings are consistent with benign schwannoma. In the night of the operation day, the patient complained of mild dyspnea and chest X-ray showed haziness in the right lung, which must have developed by re-expansion pulmonary edema after removal the mass. The patient was closely monitored in the intensive care unit. 2 days later, the pulmonary edema was resolved and the patient was transferred to the general ward. Chest X-ray taken a month after the surgery showed full expansion of right lung
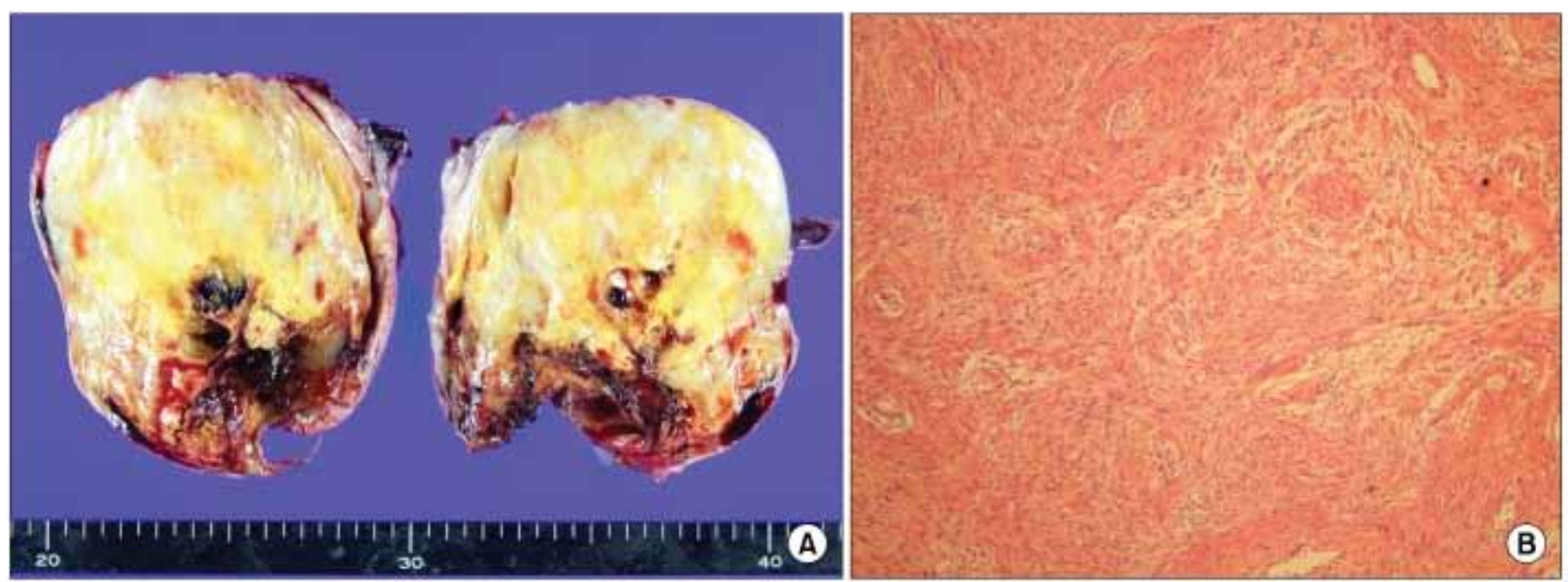

Fig-3: Pathologic examination. (A) Upon gross evaluation, the mass was a whitish soft tumor measuring $10.0 \times 12.0 \times 3.0 \mathrm{~cm}$ with a yellow cut-surface. (B) Upon microscopic exam, the tumor was composed of spindle cells with elongated nuclei, forming. 


\section{Discussion}

Neurogenic tumors comprise $19 \%$ to $39 \%$ of all mediastinal tumors. They develop from mediastinal peripheral nerve, sympathetic and parasympathetic ganglia, and embryonic remnants of neural tube. Because posterior compartment of mediastinum includes spinal nerves, vagus nerve, and sympathetic chains, neurogenic tumors of the mediastinum are most commonly present in the posterior mediastinal compartment. Among posterior mediastinal neurogenic tumors, schwannoma is the most common. Mediastinal benign schwannomas originate from Schwann cells. They affect both genders equally and develop predominantly in the third and fourth decades of life. Multiple tumors can be presented with neurofibromatosis. Mediastinal benign schwannomas are most often asymptomatic. However, sometimes mediastinal benign schwannomas can cause severe problems such as cardiac tamponade or pleural effusion, though bloody pleural effusion is usually associated with malignant schwannoma. Benign schwannomas are typically treated by surgical resection. VATS can decrease hospital stay and minimize post-operative complications; it has become the preferred method for resection for posterior neurogenic tumors. In the present case, we performed open thoracotomy because the mass was large and fixed to the posterior thoracic wall. It is important to note that postoperative course may be complicated in schwannoma cases by

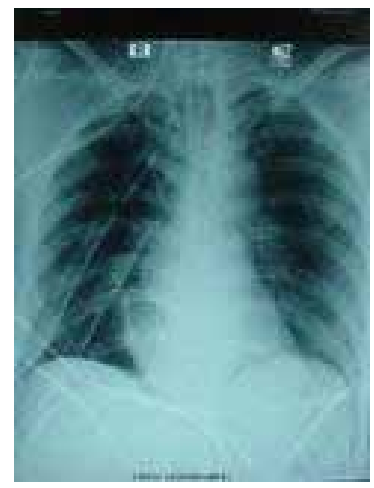

Fig: Chest X-ray P/A view after removal of the the mass. massive pleural effusion. Our patient suffered from re-expansion pulmonary edema after removal of a large mass and but no pleural effusion.

\section{Summary}

Though sometime benign schwannoma can be accompanied by massive, bloody pleural effusion but most often asymptomatic and incidental radiological diagnosis are common. However, re-expansion pulmonary edema may occur routinely.

Schwannomas are usually identified only after surgical removal. While magnetic resonance imaging (MRI) scans are the best at defining the borders and size of a tumor within a nerve, it rarely differentiates between a neurofibroma and a schwannoma.

If the schwannoma is small and asymptomatic, regular scanning and periodic check-ups is recommended. Surgery is indicated if the symptoms of the tumor are interfering with quality of life, if the tumor is growing rapidly or malignancy is suspected.

\section{References}

1. Shao J, Zhu XH, Shi JY, Ma J, Ge XJ, You ZQ. Primary pulmonary schwannoma: clinical analysis of 7 cases and review of the literature. Zhonghua Jie $\mathrm{He} \mathrm{He} \mathrm{Hu} \mathrm{Xi} \mathrm{Za}$ Zhi. 2003;26:3-6.

2. Marchevsky AM. Mediastinal tumors of peripheral nervous system origin. Semin Diagn Pathol. 1999;16:65-78.

3. Kato M, Shiota S, Shiga K, Takagi H, Mori H, Sekiya M, et al. Benign giant mediastinal schwannoma presenting as cardiac tamponade in a woman: a case report. J Med Case Rep. 2011;5:61.

4. Hazelrigg SR, Boley TM, Krasna MJ, Landreneau RJ, Yim AP. Thoracoscopic resection of posterior neurogenic tumors. Am Surg. 1999;65:1129-1133.

5. Ju Young Jang, Jin Se Kim, Ju Won Choe, Mi Kyung Kim, Jae Woo Jung et al. A case of giant benign schwannoma associated with total lung collapse by bloody effusion. Tuberc Respir Dis Seoul. 2013 August;75(2):71-74.

6. Fatimi SH, Bawany SA, Ashfaq A. Ganglioneuroblastoma of the posterior mediastinum: a case report. J Med Case Rep. 2011;5:322. 